\title{
TeraGrid: A Foundation for US Cyberinfrastructure
}

\author{
Charles E. Catlett \\ Senior Fellow, Computation Institute of Argonne \\ National Laboratory and University of Chicago \\ cec@uchicago.edu
}

\begin{abstract}
TeraGrid is a collaboration of partners providing a high-performance, nationally distributed capability infrastructure for computational science. The TeraGrid team has utilized multiple surveys of user requirements to develop five-year roadmaps describing new capabilities and services, organized into several new initiatives: Deep, Wide, and Open. TeraGrid is managed by the University of Chicago and includes resources at eight partner sites (Argonne National Laboratory, Indiana University, National Center for Supercomputing Applications, Oak Ridge National Laboratory, Pittsburgh Supercomputing Center, Purdue University, San Diego Supercomputer Center, and Texas Advanced Computing Center).

TeraGrid Deep aims to assist scientists with applications that require the combination of multiple leadership class systems- including TeraGrid storage, computing, instruments, visualization, etc. - working in concert. A team of roughly 15 staff is providing hands-on assistance to application teams pursuing TeraGrid Deep projects.

TeraGrid Wide is a set of partnerships with peer Grid projects and prototype "science gateways" that are aimed at making TeraGrid resources available to, and tailored to, entire communities of users. Science gateways are driving policy, process, and technology standards to enable web portals, desktop applications, campus clusters, and other grid infrastructure projects to seamlessly use TeraGrid resources. Initial TeraGrid science gateway projects include community portals and desktop tools supporting life sciences and biomedicine, high-energy physics, neutron science, astronomy, nanotechnology, atmospheric and climate sciences, and environmental and emergency decisionsupport.

TeraGrid Open involves the rapid evolution of the TeraGrid software and services toward interoperability with peer Grids and campus resources. Currently TeraGrid is partnering with the Open Science Grid as well as partners in Europe (e.g. UK eScience, DEISA) and Asia-Pacific (e.g. Naregi, $\mathrm{K}^{*}$ Grid).
\end{abstract}

